\section{Recurrent Abdominal Pain in Chilaiditi's Syndrome Could Represent an Indication for Elective Surgery?}

A 63-year-old male patient with hepatodiaphragmatic interposition of the intestine was admitted in our department due to chronic recurrent abdominal pain of 5 years duration. A diagnosis of Chilaiditi's sign was made as an incidental finding during a staging of nonHodgkin lymphoma, which completely healed when the patient was 47 years-old. He had not complained of any symptoms related to this condition until the age of 58 years when he started to report nausea, vomiting, constipation, and recurrent abdominal pain more intense in the right upper abdominal quadrant occurring at intervals of about 2 weeks, associated with dyspnea and episodic retrosternal pain that were more pronounced when he was lying on his left side and supine position.

Laboratory data, cardiologic and respiratory investigations, and a complete gastroenterological workup, performed to exclude causes of intermittent non surgical abdominal pain, resulted normal. A CT abdominal scan confirmed the interposition of the hepatic flexure of the colon between the liver and the diaphragm and a severe hypotropia of the right liver lobe, especially in the IV segment (Fig. 1). Due to a failure of conservative therapy, surgery was advised. At the laparoscopic exploration, the hypotrophia of IV segment of the liver associated with the absence of the hepatic flexure suspensory ligament were detected. The hepatic flexure and the first part of the transverse colon were distended and subsided in the right subphrenic space. After the dissection of the adhesions, the colon was mobilized from its abnormal position and a colopexy was performed fixing it to the parietal peritoneum of the abdominal wall with nonabsorbable interrupted sutures. The postoperative course was uncomplicated and the patient was discharged 24 hours after the operation. At 36 months follow-up the patient remained asymptomatic without medical therapy and a chest X-ray showed a normal finding.

Hepatodiaphragmatic interposition of the intestine was described for the first time in 1865 and afterwards in 1910 , by a radiologist, Demetrio Chilaiditi. It represents an incidental finding during chest or abdominal radiographs with an incidence varying from 0.14 per cent to 0.25 per cent and occurs most often in the elderly and in the male gender. ${ }^{1}$ This condition

Address correspondence and reprint requests to Prof. Gaetano Di Vita, M.D., Director, General Surgery Unit, Department of Surgical and Oncological Science, University of Palermo, Italy, Via Liborio Giuffrè $\mathrm{n}^{\circ}$ 5, 90100 Palermo, Italy. E-mail: divitagaetano@libero.it.

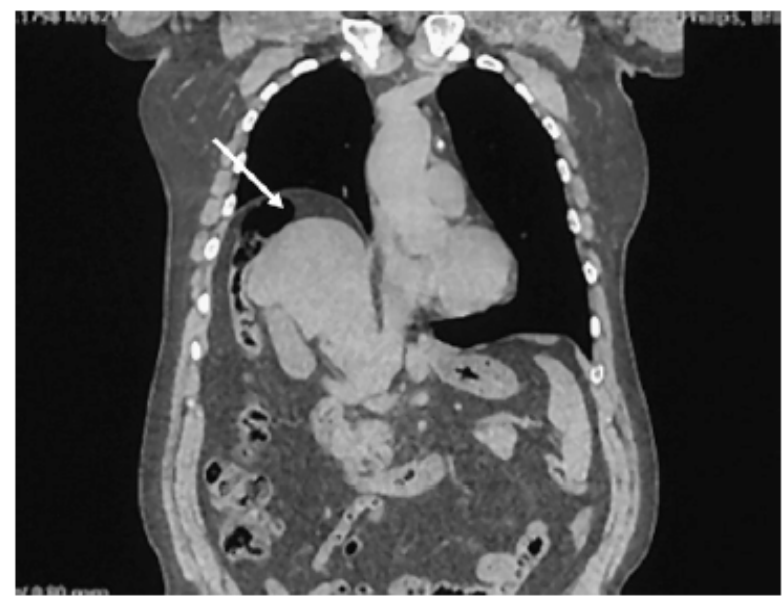

FIG. 1. Abdominal CT scan showing the interposition of the colon between the liver and the right hemidiaphragm and the hypotrophia of the IV segment of the liver.

(Chilaiditi's sign) is usually asymptomatic. Chilaiditi's syndrome (CHsy) occurs when this abnormal position of the bowel produces symptoms. The interposed bowel is usually transverse colon or hepatic flexure, but examples of interposed small bowel loops have been reported. The clinical presentation of CHsy is various. The symptoms can be referred to digestive tract and present with abdominal pain, nausea, anorexia, vomiting, and abdominal distension; to respiratory tract arising with dyspnea, respiratory distress; or they can be manifesting as cardiac affections with arrhythmias or angina like retrosternal pain. ${ }^{1}$ Due to a wide range of clinical signs, the true prevalence of CHsy in the general population cannot be precisely estimated. However, the case of CHsy complicated by bowel obstruction, volvulus of the colon, fever, and peritoneal sign up to bowel ischemia that requires an emergency surgical operation are different. ${ }^{2}$ Among the possible factors that have been implicated in the pathogenesis of this disease, it is possible to encounter intestinal factors including redundant colon with a long mesentery associated to the absence of peritoneal attachment and abnormal colon mobility; diaphragmatic factors like eventration or phrenic nerve injury; and hepatic factors including small liver caused by cirrhosis, relaxation of the suspensory ligament, and segmental agenesis of the liver, as in our case. Other factors that can play a role in the pathogenesis of this disease may be obesity and previous surgery. The diagnosis of CHsy in the majority of cases is obtained by an erect abdominal play film or chest X-ray. Abdominal ultrasound also shows the presence of an amorphous echo pattern in the hepatodiaphragmatic space. A CT scan is recommended to better define the presence of the bowel between the liver and the diaphragm and also to evidence the 
possible associated anatomic alterations. Due to a variety of clinical pictures, different clinical instrumental investigations are needed to exclude other diseases. The treatment of CHsy is usually conservative, and in the majority of cases, is sufficient alone to determine a complete resolution of symptoms. It consists of bed rest, fluid supplementation, high fiber diet, stool softeners, and drugs that reduce the gaseous distension. ${ }^{1}$ To date, there are only six cases of CHsy without complications treated surgically after failure of conservative therapy. ${ }^{3}$ In every case, the patients complained of recurrent abdominal pain and after the surgery, were symptom free, even though there are not uniform data about the type of surgical repair. Some authors freed the adherent colon from its abnormal position and fixed it to the parietal peritoneum at the level of the umbilicus. Others used the hepatopexy in which the divided falciform ligament is sutured to the right costal border. Recently, Altomare et al. ${ }^{4}$ performed anterior colopexy of the right flexure and transverse colon to the anterior abdominal wall. Therefore, the majority of cases were conducted with traditional laparotomy. To our knowledge, in fact, only two cases of laparoscopic management of CHsy have been described in the literature before now. However, in cases of severe presentation with complications like intestinal obstruction, volvulus, or ischemia, surgical intervention becomes mandatory and consists of intestinal resection of various extension. ${ }^{2}$

Actually, due to a wide range of clinical presentation, there are not signs or symptoms that indicate when CHsy will be complicated. However, it emerges that the patients affected by $\mathrm{CHsy}$, who required an emergency surgical operation, complained of a long history of ab- dominal pain associated with various episodes of intestinal subobstruction due to volvulus that required hospitalization. ${ }^{3}$ In the majority of cases, the patients were treated before surgery with nasogastric or colonoscopic decompression. Therefore, it is known that simple reduction of volvulus leaves a potential for recurrence and its decompression is generally unsuccessful. Because of the above considerations, we retain that an elective surgical operation should be considered in every patient who presented with a long history of recurrent abdominal pain with intermittent intestinal subobstruction to avoid the occurrence of ischemic complications that require emergency surgical treatment. Laparoscopic colopexy, for its reduced invasiveness and good clinical outcome, could especially represent one of the best surgical options.

Patti Rosalia, M.D., Ph.D. Territo Valentina, M.D. Gaetano Di Vita, M.D.

University of Palermo

Palermo, Italy

\section{REFERENCES}

1. Plorde JJ, Raker EJ. Transverse colonic volvulus and associated Chilaiditi's syndrome: case report and literature review. Am J Gastroenterol 1996;91:2613-6.

2. Havenstrite KA, Harris JA, Rivera DE. Splenic flexure volvolus in association with Chilaiditi syndrome: report of a case. Am Surg 1999;65:874-6.

3. Saber AA, Boros MJ. Chilaiditi's syndrome: what should every surgeon know? Am Surg 2005;71:261-3.

4. Altomare DF, Rinaldi M, Petrolino M, et al. Chilaiditi's syndrome. Successful surgical correction by colopexy. Tech Coloproctol 2001;5:173-5. 